\title{
Significant Therapeutic Efficacy with Combined Radioimmunotherapy and Cetuximab in Preclinical Models of Colorectal Cancer
}

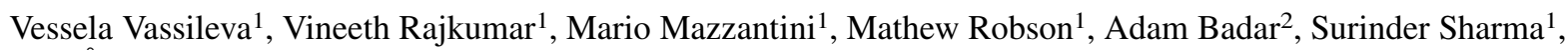 \\ Erik Årstad ${ }^{3}$, Daniel Hochhauser ${ }^{1}$, Mark F. Lythgoe ${ }^{2}$, Geoffrey M. Boxer ${ }^{1}$, and Rosmund Barbara Pedley ${ }^{1}$ \\ ${ }^{I}$ Department of Oncology, UCL Cancer Institute, University College London, London, United; Kingdom; ${ }^{2}$ Centre for Advanced \\ Biomedical Imaging (CABI), Division of Medicine, University College London, London, United Kingdom; and ${ }^{3}$ Department of \\ Chemistry and Institute of Nuclear Medicine, University College London, London, United Kingdom
}

\begin{abstract}
Despite extensive efforts to improve the clinical management of patients with colorectal cancer, approved treatments for advanced disease offer limited survival benefit. Therefore, the identification of novel treatment strategies is essential. We evaluated the preclinical efficacy of combination radioimmunotherapy, using a humanized ${ }^{131} \mathrm{I}$ labeled anti-carcinoembryonic antigen antibody ( ${ }^{131} \mid$-huA5B7), with cetuximab in colorectal cancer (CRC). Methods: Three human CRC cell lines-SW1222, LoVo, and LS174T-were used to generate subcutaneous xenografts, and stably luciferase-transfected SW1222 cells were used to establish a model of hepatic metastases in immunocompromised mice. Imaging and biodistribution studies were conducted to confirm the selective tumor localization of ${ }^{131}$ I-huA5B7. Efficacy was evaluated on the basis of tumor growth delay and survival, along with markers of DNA damage response, cell cycle, proliferation, and apoptosis. Results: Selective tumor targeting was achieved with ${ }^{131}$ IhuA5B7 alone or in combination with cetuximab without observable toxicity. Compared with monotherapy, combining cetuximab with radioimmunotherapy significantly and synergistically reduced tumor growth and prolonged survival of mice in 2 of the subcutaneous and in the metastatic tumor model. Evidence of DNA damage, G2/M arrest, significantly decreased proliferation, and increased apoptosis were observed with radioimmunotherapy and the combination therapy. However, a significant decrease in DNA-protein kinase expression with the combination regimen suggests that the addition of cetuximab suppressed DNA repair. Conclusion: Our results demonstrate enhanced therapeutic efficacy with the combination of cetuximab and radioimmunotherapy in CRC, which could potentially translate into successful clinical outcomes. This strategy could improve the treatment of residual disease postoperatively and ultimately prevent or delay recurrence. Furthermore, other carcinoembryonic antigen-expressing malignancies could also benefit from this approach.
\end{abstract}

Key Words: radioimmunotherapy; colorectal cancer; combination therapy; radiosensitization; anti-EGFR therapy

J Nucl Med 2015; 56:1239-1245

DOI: 10.2967/jnumed.115.157362

\footnotetext{
Received Mar. 10, 2015; revision accepted May 19, 2015.

For correspondence or reprints contact: Vessela Vassileva, UCL Cancer Institute, 72 Huntley St., Paul O'Gorman Bldg., London, United Kingdom WC1E 6BT.

E-mail: vessie_vassileva@hotmail.com

Published online Jun. 4, 2015.

COPYRIGHT (c) 2015 by the Society of Nuclear Medicine and Molecular Imaging, Inc.
}

C olorectal cancer (CRC) is the third most commonly diagnosed malignancy and the fourth leading cause of cancer-related deaths worldwide $(1,2)$. More than $20 \%$ of patients are diagnosed with advanced metastatic disease, with a 5-y survival of less than $10 \%$. Despite extensive efforts to improve the clinical management of patients with $\mathrm{CRC}$, approved treatments offer limited survival benefit; therefore, alternative therapeutic strategies need to be explored.

Radiotherapy has an important role in controlling local disease, increasing the possibility of resection and extending survival; however, it cannot achieve systemic reduction of metastatic disease. The use of more-targeted treatments, such as radioimmunotherapy, provides the opportunity to treat localized, metastatic, diffuse, and occult disease. Radioimmunotherapy can selectively target tumor cell- or tumor microenvironment-associated antigens with radiolabeled antibodies, delivering the greatest dose of ionizing radiation (IR) to tumor sites, while minimizing toxicity to normal tissues (35). We, and others, have previously shown that radioimmunotherapy against carcinoembryonic antigen (CEA) produced response in patients with advanced CRC; however, although some patients experienced partial remission or disease stabilization, most had disease progression (6-9). Radioimmunotherapy has been successfully used for the treatment of lymphoma, but its use in solid tumors remains to be optimized. Radioresistance, low tumor uptake, and heterogeneous distribution of radiolabeled antibodies are contributing factors to the limitations of radioimmunotherapy. To overcome these challenges, effective combination strategies that could have synergistic or additive effects with minimal toxicity should be investigated.

IR can damage DNA by producing single- and double-strand breaks (10). IR-induced DNA damage activates several signaling cascades that control cell cycle and DNA repair. Double-strand breaks are the most lethal form of damage, and inefficient repair and accumulation of double-strand breaks leads to chromosome aberrations that kill cells through apoptosis or mitotic death. Inhibition of the DNA damage response and repair is a promising strategy for sensitizing cancer cells to the lethal effects induced by IR.

The epidermal growth factor receptor (EGFR) is overexpressed in a variety of tumors, including in $60 \%-80 \%$ of CRCs, and is directly implicated in disease initiation and progression, resistance to therapy, and poor prognosis (11). IR induces the nuclear translocation of EGFR, where it associates with the catalytic subunit of DNA-dependent protein kinase (DNA-PKcs), stimulating the repair of double-strand breaks. 
The use of EGFR inhibitors, such as cetuximab, hinders DNA repair by blocking the nuclear translocation of EGFR and hence increases the sensitivity of cells to ionizing radiation (12). The ability of cetuximab as a radiosensitizer has been demonstrated in head and neck cancer $(13,14)$ and in vitro in CRC cell lines (15). To our knowledge, there are no reports that have specifically addressed the combination of cetuximab with radioimmunotherapy in CRC. The main objective of this study was to assess the effect of combining cetuximab with radioimmunotherapy, using a humanized ${ }^{131}$ I-labeled anti-CEA antibody ( ${ }^{131} \mathrm{I}$-huA5B7), in preclinical models of CRC.

\section{MATERIALS AND METHODS}

\section{Agents and Antibody Labeling}

Cetuximab (Erbitux [Merck], $5 \mathrm{mg} / \mathrm{mL}$ solution) was purchased from the University College London Hospital Pharmacy. HuA5B7 was provided by UCB Pharma S.A. and labeled with ${ }^{131} \mathrm{I}\left({ }^{131} \mathrm{I}\right.$-huA5B7) (Perkin Elmer, U.K.) using the IODO-GEN (Pierce) method as previously described $(16,17)$. Labeling yield and radiochemical purity were assessed by thin-layer chromatography (silica gel Si60, stationary phase; 80\% methanol, mobile phase); antigen binding of the radiolabeled antibody was compared with nonlabeled huA5B7 and assessed by enzyme-linked immunosorbent assay.

\section{Cell Lines}

Human colorectal adenocarcinoma cell lines SW1222, LoVo, and LS174T were obtained from the European Collection of Cell Cultures. All 3 cell lines are reported to have KRAS mutations (A146V, G13D/ $\mathrm{A} 14 \mathrm{~V}$, and G12D, respectively) $(18,19)$. Cells were maintained in Dulbecco modified Eagle medium (Invitrogen) supplemented with $10 \%$ fetal bovine serum (Invitrogen) at $37^{\circ} \mathrm{C}$ under humidified atmosphere containing $5 \% \mathrm{CO}_{2}$.

\section{Tumor Models}

Female nude CD-1 and MF-1 mice (age, 6-8 wk; weight, 20-25 g) were purchased from Charles River and Harlan Laboratories, respectively. All animal experiments were performed in accordance with the U.K. Home Office Animals Scientific Procedures Act 1986 and United Kingdom Co-ordinating Committee on Cancer Research Guidelines for the Welfare and Use of Animals in Cancer Research (20) and approved by the University College London Animal Welfare and Ethical Review Body under project license 70-309.

Subcutaneous xenografts were established in CD-1 nude mice using SW1222, LoVo, or LS174T cells. Briefly, $5 \times 10^{6}$ cells were injected into the right flank of mice and allowed to grow to a mean volume of $0.1 \mathrm{~cm}^{3}$. Tumor volumes were caliper-measured and calculated using the formula volume $=4 \pi / 3$ (1/2 length $\times 1 / 2$ width $\times 1 / 2$ height $)$. Mice were sacrificed when tumors reached $1.5 \mathrm{~cm}^{3}$ (endpoint).

Orthotopic models of hepatic metastases were generated in MF-1 nude mice using SW1222 cells transfected with the luciferase gene (SW1222 Luc cells). Briefly, $2 \times 10^{6}$ cells were injected into the spleen, followed by a splenectomy after $10 \mathrm{~min}(21,22)$. Hepatic tumor growth was monitored by bioluminescence imaging using the IVIS system (Xenogen/Caliper Life Sciences) as previously described (23). The Living Image Software (Xenogen/Caliper Life Sciences) was used to obtain and analyze images. Regions of interest were created and the counts per second measured (tumor regions in each group had an average of $2.1 \times 10^{6}$ total counts/s at the start of the studies, $\sim 14 \mathrm{~d}$ after tumor cell inoculation).

\section{Localization and Biodistribution of ${ }^{131}$ I-huA5B7}

Localization and biodistribution of ${ }^{131}$ I-huA5B7 was assessed with and without cetuximab, at 2, 6, and $10 \mathrm{~d}$ after administration in the subcutaneous tumor models and at $2 \mathrm{~d}$ in the metastatic tumor model. Briefly, mice ( $n=4$, per group) were injected intravenously with $1 \mathrm{MBq}\left({ }^{131} \mathrm{I}-\mathrm{huA}\right.$ 5B7, $0.25 \mu \mathrm{g}$, in total volume of $100 \mu \mathrm{L}$ of sterile saline) with or without cetuximab $(10 \mathrm{mg} / \mathrm{kg})$ administered intraperitoneally $4 \mathrm{~h}$ before radioimmunotherapy and imaged using SPECT/CT (Silver Upgrade NanoSPECT/CT; Mediso) at the selected time points. Mice were then euthanized, and tissues (blood, liver, kidney, lung, spleen, colon, muscle, tumor) were collected, weighed, and $\gamma$-counted (WIZARD; PerkinElmer, U.K.). The uptake of ${ }^{131}$ I-huA5B7 was calculated as percentage injected activity per gram of tissue.

\section{Efficacy Studies}

Efficacy studies were performed in the subcutaneous SW1222, LoVo, and LS174T cell lines and in the metastatic tumor model. Mice with similar tumor burden were allocated to the following groups, $n=8$ per group: untreated, cetuximab, ${ }^{131} \mathrm{I}$-huA5B7, and cetuximab combined with ${ }^{131} \mathrm{I}$-huA5B7. Cetuximab $(10 \mathrm{mg} / \mathrm{kg})$ was administered intraperitoneally twice weekly, radioimmunotherapy consisted of a single intravenous injection of ${ }^{131} \mathrm{I}$-huA5B7 (1 MBq), and the combined treatment involved administration of cetuximab $4 \mathrm{~h}$ before ${ }^{131} \mathrm{I}$-huA5B7 and twice weekly as maintenance therapy. Tumor volumes were measured, and mice were weighed every 2-3 d throughout the duration of the study until endpoint. In the metastatic model, tumor burden was assessed by bioluminescence imaging as described above; mice were imaged every $2-3 \mathrm{~d}$ to monitor tumor growth over time. Disease progression was also evaluated qualitatively by observation of behavior, weight loss, muscle wasting, and abdominal distension, as previously described $(24,25)$. Animals were sacrificed when a signal of greater $2 \times 10^{7}$ total counts/s was measured, which correlated with the qualitative measurements of disease progression.

Efficacy with various activities $(0.5,1,1.5$, and $2 \mathrm{MBq})$ of ${ }^{131} \mathrm{I}$-huA5B7 $(0.25 \mu \mathrm{g}$, in total volume of $100 \mu \mathrm{L}$ of sterile saline) alone or in combination with cetuximab was assessed in the subcutaneous SW1222 model.

Tumor growth curves based on changes in tumor volume or bioluminescence over time and survival were used to evaluate treatment efficacy.

\section{Immunohistochemistry}

CEA expression was confirmed in all tumor models as previously described (16). Markers of DNA damage response ( $\gamma \mathrm{H} 2 \mathrm{AX}$ and DNA$\mathrm{PK}$ ), cell cycle (phospho-histone H3, cyclin B1), proliferation (Ki-67), and apoptosis (caspase-3) were examined in tumor sections from each group at 2, 6, and $10 \mathrm{~d}$ after treatment initiation. After antigen retrieval, sections were incubated with primary antibodies either for $1 \mathrm{~h}$ at room temperature for cyclin B1 (Abcam, 1:200), phospho-histone H3 (Millipore, 1:200), and Ki-67 (Dako, 1:300) or overnight at $4^{\circ} \mathrm{C}$ for $\gamma$-H2AX (Millipore, 1:500), DNA-PK (Abcam, 1:500), and cleaved caspase-3 (Cell Signaling, 1:500) and visualized using biotin-conjugated secondary antibodies (Vectastain ABC Kit; Vector Labs) and 3, 3'-diaminobenzidine (DAB; Dako). Sections were then counterstained with hematoxylin, dehydrated, and mounted with DPX (Sigma Aldrich).

\section{Image Analyses and Quantification}

Image acquisition and analysis were performed using the TissueGnostics software (microscope, AxioImager Z.2; Zeiss). Briefly, regions of interest were used to select viable tumor tissue and excluded necrotic areas. Cell-based analysis was performed with automated cell segmentation based on color: hematoxylin in the blue and DAB in the red channel. To avoid artifacts, a threshold for minimum cell area and variance of hematoxylin staining was set. Positive cells were selected on the basis of mean and maximum intensity of DAB. Data were generated by calculating the percentage of the DAB-reactive cells in relation to the total number of cells in the regions of interest.

\section{Combination Analyses}

The Bliss independence model was used to calculate whether the combination therapy was synergistic. Briefly, this method compares the observed response of the combination with the predicted response, which was obtained using the assumption that there is no effect from drug-drug 


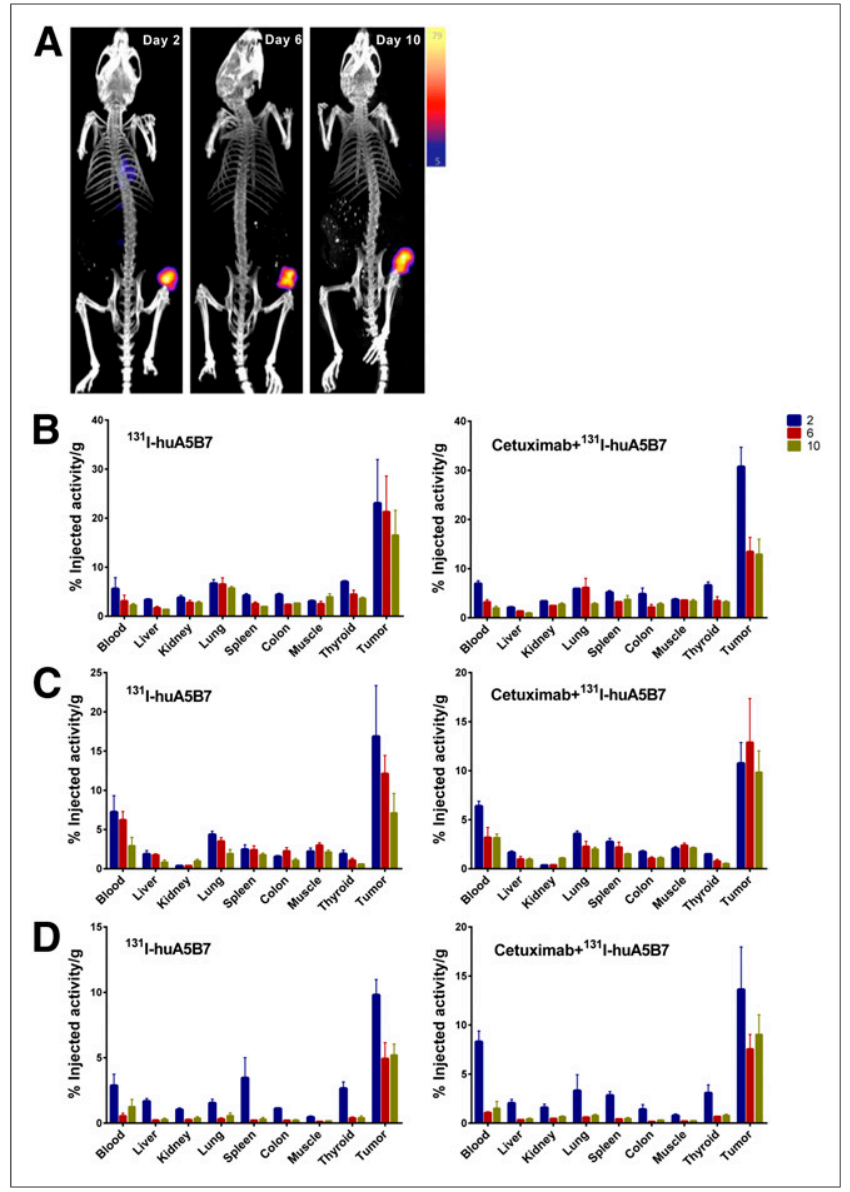

FIGURE 1. Localization and biodistribution of ${ }^{131} \mid$-huA5B7 in subcutaneous tumor xenografts. (A) Representative whole-body SPECT/CT images from SW1222 tumor model. Color bar represents counts per second with maximum and minimum threshold levels. Biodistribution of ${ }^{131}$ I-huA5B7 alone or in combination with cetuximab in tissues at 2, 6 , and $10 \mathrm{~d}$ after administration in SW1222 tumor model (B), LoVo tumor model (C), and LS174T tumor model (D) is shown. Data presented as mean percentage injected activity per gram of tissue \pm SE.

interactions (26). Typically, the combination effect is declared synergistic if the observed response is greater than predicted response.

\section{Statistical Analyses}

Data were plotted and analyzed using Prism software (version 6.0; GraphPad Software). Differences in tumor growth and immunohistochemistry markers between the groups were assessed by 2-way ANOVA and multiple $t$ tests. Kaplan-Meier survival plots were analyzed using the log-rank test. Results were considered statistically significant at a $P$ value of 0.05 or less.

\section{RESULTS}

Localization and Biodistribution of ${ }^{131}$ I-huA5B7 and Efficacy of Combination Therapy in Subcutaneous Xenografts

Whole-body SPECT/CT of tumor-bearing mice demonstrated highly selective tumor localization of ${ }^{131}{ }^{1}$-huA5B7 at 2, 6, and $10 \mathrm{~d}$ after administration (Fig. 1A). Prolonged and high tumor retention of ${ }^{131}$ I-huA5B7 was observed, compared with normal tissues (Figs. 1B-1D). The addition of cetuximab did not significantly alter the biodistribution of ${ }^{131} \mathrm{I}$-huA5B7.

Overall, the combination therapy significantly delayed tumor growth and prolonged survival, compared with each monotherapy, in the SW1222 and LoVo tumor model but not in the LS174T xenograft (Fig. 2).

Compared with untreated mice, radioimmunotherapy significantly reduced tumor growth and prolonged survival in all 3 tumor models, whereas cetuximab had an effect in the SW1222 and LoVo xenografts. The median survival of untreated, cetuximab, ${ }^{131} \mathrm{I}-\mathrm{hu} \mathrm{A} 5 \mathrm{~B} 7$, and combination-treated mice for all 3 tumor models is displayed in Table 1. No observable toxicities were detected in any of the groups from all studies.

\section{Localization and Biodistribution of ${ }^{131} \mathrm{I}$-huA5B7 and Efficacy} of Combination Therapy in Model of Hepatic Metastases

Similar to our findings in the subcutaneous tumor models, SPECT/ CT imaging of tumor-bearing mice and phosphor images of liver sections with tumor deposits showed selective tumor localization of ${ }^{131}$ I-huA5B7, $2 \mathrm{~d}$ after administration (Fig. 3A). The combination therapy significantly improved response, compared with each monotherapy, based on tumor growth delay as evaluated by bioluminescence imaging and survival analyses (Figs. 3B and 3C). Compared with untreated mice, each monotherapy also significantly delayed tumor growth and prolonged survival (Figs. 3B and 3C). The median survival of untreated, cetuximab, ${ }^{131} \mathrm{I}$-huA5B7, and combinationtreated mice was $12.5,14,18$, and $27 \mathrm{~d}$, respectively.

\section{Effect of Increasing Activity of Radioimmunotherapy Alone or in Combination with Cetuximab in SW1222 \\ Subcutaneous Model}

The studies with a range of activities of ${ }^{131} \mathrm{I}$-huA5B7 $(0.5,1$, 1.5 , and $2 \mathrm{MBq}$ ) demonstrated that the addition of cetuximab to

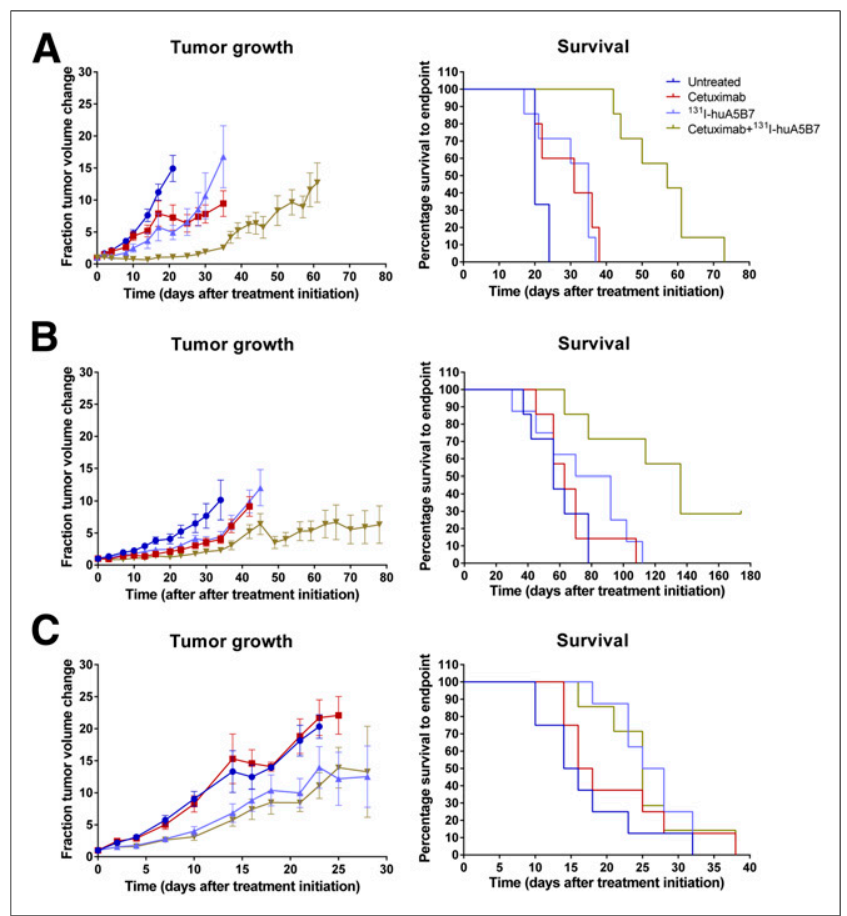

FIGURE 2. Efficacy of cetuximab, ${ }^{131}$ I-huA5B7, and combination therapy evaluated as tumor growth and survival in subcutaneous tumor models SW1222 (A), LoVo (B), and LS174T (C). Tumor growth presented as mean fraction tumor volume change over time $\pm \mathrm{SE}$, and survival data presented as Kaplan-Meier plots. Combination therapy significantly delayed tumor growth and prolonged survival, compared with each monotherapy in SW1222 and LoVo tumor model $(P<0.05$, 2-way ANOVA, multiple $t$ tests, and log-rank test). 
TABLE 1

Median Survival of Mice Treated with $1 \mathrm{MBq}$ of Radioimmunotherapy

\begin{tabular}{|c|c|c|c|}
\hline \multirow[b]{2}{*}{ Therapy } & \multicolumn{3}{|c|}{$\begin{array}{c}\text { Tumor model (median } \\
\text { survival }[\mathrm{d}] \text { ) }\end{array}$} \\
\hline & SW1222 & LoVo & LS174T \\
\hline Untreated & 20 & 56 & 15 \\
\hline Cetuximab & 31 & 63 & 17 \\
\hline${ }^{131}$ I-huA5B7 & 35 & 81 & 26.5 \\
\hline Cetuximab + ${ }^{131}$ I-huA5B7 & 57 & 136 & 25 \\
\hline
\end{tabular}

Tumor model refers to subcutaneous xenograft.

radioimmunotherapy significantly enhanced response, compared with each corresponding treatment with radioimmunotherapy alone (Fig. 4).

No significant difference in response was observed between the radioimmunotherapy-alone groups and also between the combinationtherapy groups, except with $0.5 \mathrm{MBq}$.

The response to the combination therapy with $0.5 \mathrm{MBq}$ was not significantly different from the response with $2 \mathrm{MBq}$ of radioimmunotherapy alone, demonstrating that significantly lower activity of radioimmunotherapy can be used in combination with cetuximab to achieve similar effects. Combination therapy with 1 or $1.5 \mathrm{MBq}$ significantly delayed tumor growth and prolonged survival, compared with $2 \mathrm{MBq}$ of radioimmunotherapy alone.

The median survival of untreated and cetuximab-treated mice was 20 and $35 \mathrm{~d}$. For mice treated with $0.5,1.0,1.5$, and $2.0 \mathrm{MBq}$, median survival was $22.5,35,36$, and $33 \mathrm{~d}$, respectively, and 29 , 57,57 , and $52 \mathrm{~d}$ for mice treated with the corresponding activity when combined with cetuximab.

\section{Effect of Therapy on DNA Damage Response, Cell Cycle, Proliferation, and Apoptosis}

Immunohistochemistry revealed that all xenografts expressed CEA. SW1222 and LS174T tumors had a relatively higher CEA expression than LoVo tumors (Supplemental Fig. 1; supplemental materials are available at http://jnm.snmjournals.org).

Markers of DNA damage response, cell cycle, proliferation, and apoptosis were examined in tumor sections from the SW1222 tumor model at 2, 6, and $10 \mathrm{~d}$ after treatment initiation. Evidence of DNA damage and repair were observed with radioimmunotherapy when administered alone or in combination with cetuximab (Fig. 5A). However, there was a significant decrease in DNA-PK at days 6 and 10 with the combination therapy, compared with radioimmunotherapy alone, suggesting that cetuximab suppressed DNA repair. Significantly decreased phospho-histone H3 and mainly cytoplasmic cyclin B1 expression suggested a G2/M arrest with radioimmunotherapy and the combination therapy, which was also accompanied by a significant decrease in proliferation and increase in apoptosis (Figs. 5B and 5C). A significant decrease in the percentage of cells undergoing mitosis and proliferation was observed with the combination therapy, compared with radioimmunotherapy at day 10 .

\section{Synergy}

The observed combined response with cetuximab and ${ }^{131} \mathrm{I}-\mathrm{huA} 5 \mathrm{~B} 7$ was significantly greater than the predicted response for the SW1222 (subcutaneous and metastatic) and the LoVo tumor models, indicating that the combination treatment was more efficacious than expected, which is representative of synergy (Supplemental Fig. 2). Synergy occurred from day 17 and 14 in the SW1222 subcutaneous and metastatic tumor model, respectively, and from day 45 in the LoVo tumor model. Moreover, synergy was also observed with the range of activities of radioimmunotherapy in combination with cetuximab, even with the lowest activity of $0.5 \mathrm{MBq}$, where it occurred from day 25 .

\section{DISCUSSION}

We have demonstrated that combining cetuximab with ${ }^{131} \mathrm{I}-$ huA5B7 significantly and synergistically improved therapeutic efficacy in subcutaneous and metastatic preclinical models of CRC. This enhanced therapeutic response was achieved without observable toxicity and with significantly lower than clinically used activities of radioimmunotherapy. To our knowledge, our study is the first to examine this combination strategy in CRC. In addition to its use
A

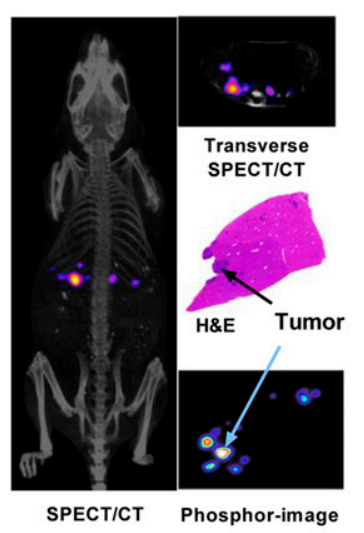

B

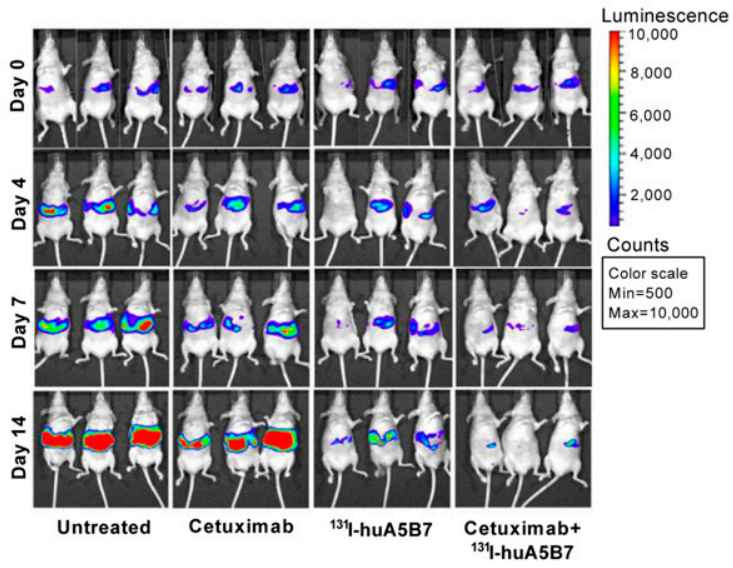

C

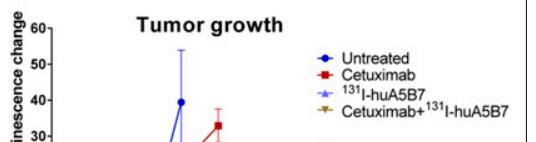

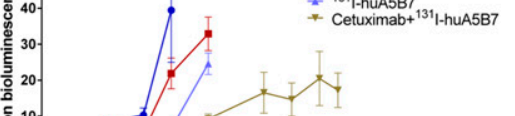
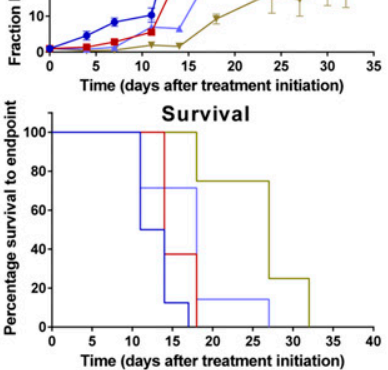

FIGURE 3. Efficacy of combination therapy in model of hepatic metastases. In vivo and ex vivo localization of ${ }^{131}$ I-huA5B7 $2 \mathrm{~d}$ after administration (A), representative bioluminescence images of 3 mice from each group (B), tumor growth represented as fractional change in bioluminescence over time $(C)$, mean \pm SE and survival of mice, Kaplan-Meier plots. Combination therapy significantly delayed tumor growth and prolonged survival, compared with each monotherapy $(P<0.05,2$-way ANOVA, multiple $t$ tests, and log-rank test). H\&E $=$ hematoxylin and eosin. 


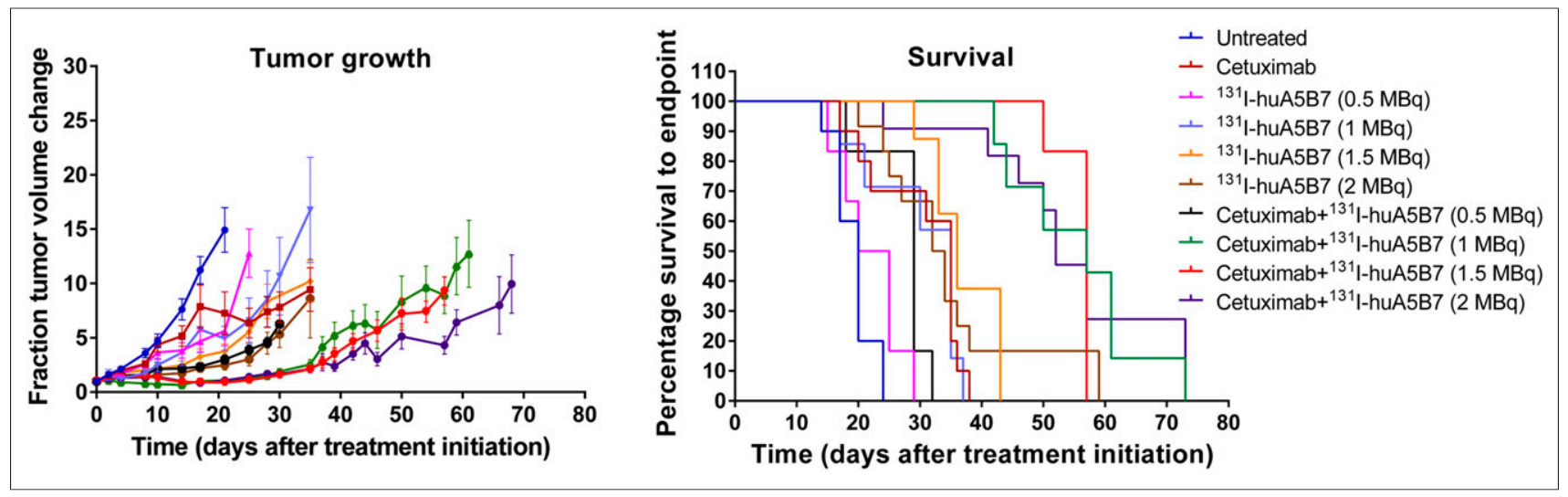

FIGURE 4. Efficacy in response to a range of activities of ${ }^{131} \mathrm{I}$-huA5B7 alone or in combination with cetuximab. Tumor growth represented as mean fraction tumor volume change over time \pm SE, and survival data presented as Kaplan-Meier plots. Addition of cetuximab to radioimmunotherapy significantly enhanced response, compared with each corresponding treatment with radioimmunotherapy alone $(P<0.05,2$-way ANOVA, multiple $t$ tests, and log-rank test).

as a therapeutic modality, radioimmunotherapy can also be simultaneously used as an imaging technique to detect the localization of the radiolabeled antibody within tumors and normal tissues.
After the confirmation of the selective localization of ${ }^{131}$ I-huA5B7 to tumor sites, the effect of the combination therapy was examined in 3 distinct subcutaneous models of CRC (SW1222, LoVo,

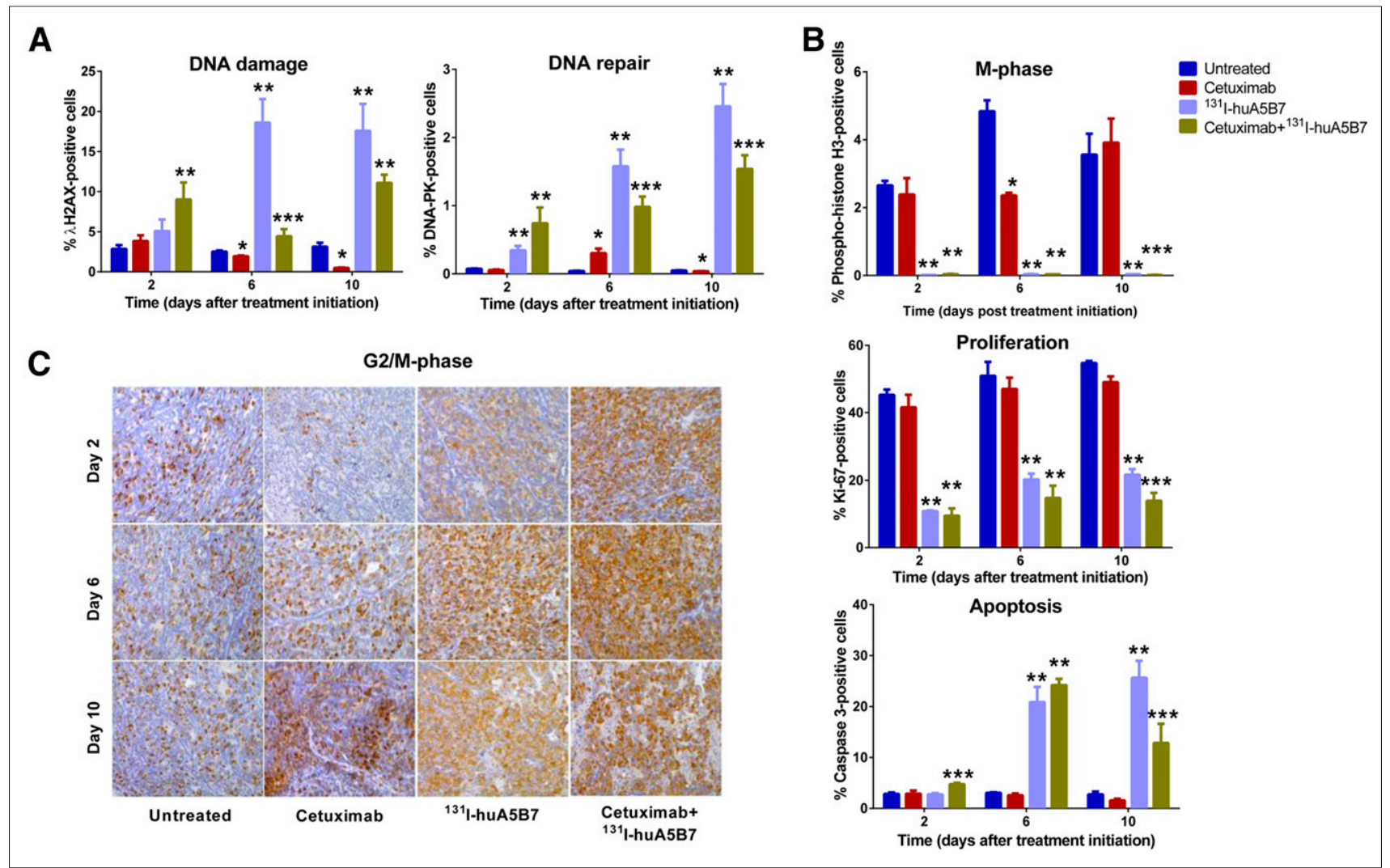

FIGURE 5. Effect of therapy in subcutaneous SW1222 xenografts on DNA damage response $(\gamma \mathrm{H} 2 \mathrm{AX}$, DNA-PK) (A); cell cycle, proliferation, and apoptosis (phospho-histone H3, Ki-67, and cleaved caspase-3) (B); and G2/M phase of cell cycle (C) (representative tumor sections from each group showing cyclin B1 distribution). Quantitative immunohistochemistry data presented as mean percentage of positive cells \pm SE. DNA damage and repair were observed with radioimmunotherapy when administered alone or in combination with cetuximab. Significant decrease in DNA-PK was detected with combination therapy at days 6 and 10, compared with radioimmunotherapy $(P<0.05$, Student $t$ test). Significantly decreased phospho-histone $\mathrm{H} 3$ and mainly cytoplasmic cyclin B1 expression were observed with radioimmunotherapy and combination therapy, which was also accompanied by significant decrease in proliferation and increase in apoptosis $(P<0.05$, Student $t$ test). *Indicates significantly different from untreated. ${ }^{*}$ Indicates significantly different from untreated and cetuximab. ${ }^{\star * \star}$ Indicates significantly different from untreated, cetuximab, and 131I-huA5B7 $(P<0.05$, ANOVA, multiple $t$ tests). 
and LS174T). Combining cetuximab with radioimmunotherapy resulted in significantly delayed tumor growth and prolonged survival, compared with each monotherapy, in both the SW1222 and the LoVo xenografts but not in the LS174 tumor model, which was responsive only to radioimmunotherapy. Clearly, the genetic variability of tumors can influence response to treatment. Although all of the 3 cell lines possess KRAS mutations, the LS174T cells harbor an additional mutation in $P I 3 C A$, which likely contributes to augmented signaling in the RAS-RAF-MEK-MAPK pathway and increased resistance to cetuximab. Although it is widely accepted that mutations in KRAS predict response to cetuximab in CRC, our data suggest that additional mutations in other genes, which contribute to augmented signaling in the RAS-RAF-MEK-MAPK or PI3K-AKT-mTOR pathways, likely lead to cetuximab resistance. Therefore, cetuximab should not be disregarded in KRAS-mutant tumors, especially when combined with radioimmunotherapy, unless they harbor additional mutations in downstream signaling pathways. Indeed, patients with CRC bearing a G12D or a G13D mutation have been described to have a more favorable outcome than other KRAS mutations, contrary to previous reports $(27,28)$.

The enhanced tumor responsiveness to the combination therapy in the SW1222 and LoVo tumor models could be as a result of selectively localized and prolonged radiation exposure and damage from radioimmunotherapy plus reduced DNA repair due to inhibition of EGFR function by cetuximab. Overall, we observed evidence of simultaneous DNA damage and repair with radioimmunotherapy alone or in combination with cetuximab. However, DNA-PK expression was lower in the combination-therapy group than in the radioimmunotherapy group, suggesting that the addition of cetuximab affected DNA repair. Even a small decrease in DNA-PK activity could result in a significantly reduced capacity of DNA repair (12). These data were also accompanied by evidence of a G2/M arrest, significantly decreased proliferation, and increased apoptosis.

Overall, our findings suggest that DNA damage occurs and accumulates with radioimmunotherapy leading to a G2/M arrest, some DNA repair, decreased proliferation, and increased apoptosis; however, the addition of cetuximab impedes DNA repair and enhances efficacy, potentially via mechanisms other than apoptosis, such as mitotic catastrophe and senescence, supported by the decreased level of mitotic and proliferating cells with the combination therapy at the latest time point. The effectiveness of the combination therapy was further confirmed in a more clinically relevant model of metastasis. Similarly to the subcutaneous xenografts, highly selective tumor localization of ${ }^{131} \mathrm{I}$-huA5B7 was observed, along with significantly enhanced tumor growth delay and survival, which was significantly enhanced by the addition of cetuximab.

We then investigated whether combining cetuximab with various activities of radioimmunotherapy could further improve therapeutic response in the SW1222 tumor model. Similar therapeutic effect was observed with all activities of radioimmunotherapy alone or when combined with cetuximab, except with $0.5 \mathrm{MBq}$. Each combination was significantly more effective than monotherapy with radioimmunotherapy or cetuximab.

Combination therapy with 1 or $1.5 \mathrm{MBq}$ significantly delayed tumor growth and prolonged survival, compared with $2 \mathrm{MBq}$ of radioimmunotherapy alone, suggesting that cetuximab is radiosensitizing tumor cells. Similarly, another study also demonstrated increased efficacy with combination therapy in preclinical models of head and neck cancer (29). However, the activities of radioimmunotherapy used in this study were in excess of what is normally administered to patients $(6,7,30)$. To our knowledge, our study is the first to demonstrate that activities as low as $1 \mathrm{MBq}$ of radioimmunotherapy can be therapeutically effective, particularly when combined with radiosensitizing agents, such as cetuximab, which would greatly facilitate the use of repeated radioimmunotherapy administration in patients. Extrapolating $1 \mathrm{MBq}$ to the human equivalent activity corresponds to approximately $154 \mathrm{MBq} / \mathrm{m}^{2}$ (31), which is significantly lower than clinically used activities. Data from an early phase I trial of single-agent ${ }^{131}$ I-murine A5B7 in patients with advanced CRC defined bone marrow suppression as the doselimiting toxicity at $2,400 \mathrm{MBq} / \mathrm{m}^{2}$, which was further reduced to $1,600 \mathrm{MBq} / \mathrm{m}^{2}$ in another trial, as a cohort of patients experienced grade 4 neutropenia $(6,7,30)$. Our findings with the humanized version of A5B7 strongly suggest that combining cetuximab with lower activity of radioimmunotherapy could translate into significantly better clinical outcomes with minimal toxicity.

Although we did not have a nonspecific radiolabeled antibody ( $\left.{ }^{131} \mathrm{I}-\mathrm{IgG} 1 / \mathrm{MOPC}\right)$ control in our studies, we have previously shown no therapeutic effect with a dose of $4 \mathrm{MBq}$ of ${ }^{131}{ }^{1}$-IgG1/ MOPC in SW1222 tumor metastases (16), suggesting that cross-fire irradiation from circulating antibody does not significantly affect tumor growth. Moreover, our current studies show that $10 \mathrm{~d}$ after administration, the tumor-to-blood ratio of huA5B7 is approximately 8:1 in SW1222 tumors with minimal blood levels, which supports the hypothesis that the observed therapeutic effects were due to tumor-bound antibody rather than circulating antibody.

\section{CONCLUSION}

We demonstrated that cetuximab synergizes with radioimmunotherapy and radiosensitizes tumor cells to significantly enhance therapeutic response in preclinical models of CRC. This strategy might represent the ideal scenario for the treatment of residual disease postoperatively and ultimately for prevention and delay of recurrence. Furthermore, KRAS-mutant tumors and other CEAexpressing malignancies could also benefit from this approach.

\section{DISCLOSURE}

The costs of publication of this article were defrayed in part by the payment of page charges. Therefore, and solely to indicate this fact, this article is hereby marked "advertisement" in accordance with 18 USC section 1734. This work was supported by MRC DPFS/DCS grant G1001497. Radiolabelling work was performed at UCLH/ UCL, partially funded by the Department of Health NIHR Biomedical Research Centers funding scheme. Imaging work was conducted in collaboration with CRUK and EPSRC Comprehensive Cancer Imaging Centre at KCL and UCL, jointly funded by Cancer Research U.K. and the Engineering and Physical Sciences Research Council (EPSRC), grant C1519/A10331 and C1519/A16463. No other potential conflict of interest relevant to this article was reported.

\section{ACKNOWLEDGMENTS}

We acknowledge Dr. Jane Kinghorn for her support. We are grateful to UCB Pharma S.A, Slough, U.K., for providing the huA5B7 antibody. We also acknowledge Mohammed Rashid and Chiea K. Cheng for their technical support.

\section{REFERENCES}

1. Curtin JC. Novel drug discovery opportunities for colorectal cancer. Expert Opin Drug Discov. 2013;8:1153-1164.

2. Siegel R, Naishadham D, Jemal A. Cancer statistics, 2013. CA Cancer J Clin. 2013;63:11-30. 
3. Navarro-Teulon I, Lozza C, Pelegrin A, Vives E, Pouget JP. General overview of radioimmunotherapy of solid tumors. Immunotherapy. 2013;5:467-487.

4. Dearling JL, Pedley RB. Technological advances in radioimmunotherapy. Clin Oncol (R Coll Radiol). 2007;19:457-469.

5. Pouget J-P, Navarro-Teulon I, Bardies M, et al. Clinical radioimmunotherapy: the role of radiobiology. Nat Rev Clin Oncol. 2011;8:720-734.

6. Lane DM, Eagle KF, Begent RH, et al. Radioimmunotherapy of metastatic colorectal tumours with iodine-131-labelled antibody to carcinoembryonic antigen: phase I/II study with comparative biodistribution of intact and F(ab')2 antibodies. Br J Cancer. 1994;70:521-525.

7. Meyer T, Gaya AM, Dancey G, et al. A phase I trial of radioimmunotherapy with ${ }^{131}$ I-A5B7 anti-CEA antibody in combination with combretastatin-A4-phosphate in advanced gastrointestinal carcinomas. Clin Cancer Res. 2009;15:4484-4492.

8. Juweid ME, Sharkey RM, Behr T, et al. Radioimmunotherapy of patients with small-volume tumors using iodine-131-labeled anti-CEA monoclonal antibody NP-4 F(ab')2. J Nucl Med. 1996;37:1504-1510.

9. Hajjar G, Sharkey RM, Burton J, et al. Phase I radioimmunotherapy trial with iodine-131-labeled humanized $\mathrm{MN}-14$ anti-carcinoembryonic antigen monoclonal antibody in patients with metastatic gastrointestinal and colorectal cancer. Clin Colorectal Cancer. 2002;2:31-42.

10. Santivasi WL, Xia F. Ionizing radiation-induced DNA damage, response, and repair. Antioxid Redox Signal. 2014;21:8.

11. Cunningham D, Humblet $\mathrm{Y}$, Siena $\mathrm{S}$, et al. Cetuximab monotherapy and cetuximab plus irinotecan in irinotecan-refractory metastatic colorectal cancer. $N$ Engl J Med. 2004;351:337-345

12. Liccardi G, Hartley JA, Hochhauser D. Importance of EGFR/ERCC1 interaction following radiation-induced DNA damage. Clin Cancer Res. 2014;20:3496-3506.

13. Bonner JA, Harari PM, Giralt J, et al. Radiotherapy plus cetuximab for squamouscell carcinoma of the head and neck. N Engl J Med. 2006;354:567-578.

14. Bonner JA, Harari PM, Giralt J, et al. Radiotherapy plus cetuximab for locoregionally advanced head and neck cancer: 5-year survival data from a phase 3 randomised trial, and relation between cetuximab-induced rash and survival. Lancet Oncol. 2010;11:21-28.

15. Shin HK, Kim MS, Lee JK, et al. Combination effect of cetuximab with radiation in colorectal cancer cells. Tumori. 2010;96:713-720.

16. El Emir E, Qureshi U, Dearling JL, et al. Predicting response to radioimmunotherapy from the tumor microenvironment of colorectal carcinomas. Cancer Res. 2007;67:11896-11905.

17. Rajkumar VS, Boxer G, Robson M, Muddle J, Papastavrou Y, Pedley RB. A comparative study of PDGFR inhibition with imatinib on radiolabeled antibody targeting and clearance in two pathologically distinct models of colon adenocarcinoma. Tumour Biol. 2012;33:2019-2029.
18. Therkildsen C, Bergmann TK, Henrichsen-Schnack T, Ladelund S, Nilbert M. The predictive value of KRAS, NRAS, BRAF, PIK3CA and PTEN for antiEGFR treatment in metastatic colorectal cancer: a systematic review and metaanalysis. Acta Oncol. 2014;53:852-864

19. Tol J, Dijkstra JR, Klomp M, et al. Markers for EGFR pathway activation as predictor of outcome in metastatic colorectal cancer patients treated with or without cetuximab. Eur J Cancer. 2010;46:1997-2009.

20. Workman P, Aboagye EO, Balkwill F, et al. Guidelines for the welfare and use of animals in cancer research. Br J Cancer. 2010;102:1555-1577.

21. Rajendran S, Salwa S, Gao X, et al. Murine bioluminescent hepatic tumour model. J Vis Exp. 2010;1977.

22. Dearling JL, Flynn AA, Qureshi U, et al. Localization of radiolabeled anti-CEA antibody in subcutaneous and intrahepatic colorectal xenografts: influence of tumor size and location within host organ on antibody uptake. Nucl Med Biol. 2009;36:883-894.

23. Vassileva V, Moriyama EH, De Souza R, et al. Efficacy assessment of sustained intraperitoneal paclitaxel therapy in a murine model of ovarian cancer using bioluminescent imaging. Br J Cancer. 2008;99:2037-2043.

24. Vassileva V, Allen CJ, Piquette-Miller M. Effects of sustained and intermittent paclitaxel therapy on tumor repopulation in ovarian cancer. Mol Cancer Ther. 2008;7:630-637.

25. Ullman-Culleré MH, Foltz CJ. Body condition scoring: a rapid and accurate method for assessing health status in mice. Lab Anim Sci. 1999;49:319-323.

26. Zhao W, Sachsenmeier K, Zhang L, Sult E, Hollingsworth RE, Yang H. A new bliss independence model to analyze drug combination data. J Biomol Screen. 2014;19:817-821.

27. De Roock W, Jonker DJ, Di Nicolantonio F, et al. Association of KRAS p.G13D mutation with outcome in patients with chemotherapy-refractory metastatic colorectal cancer treated with cetuximab. JAMA. 2010;304:1812-1820.

28. Modest DP, Brodowicz T, Stintzing S, et al. Impact of the specific mutation in KRAS codon 12 mutated tumors on treatment efficacy in patients with metastatic colorectal cancer receiving cetuximab-based first-line therapy: a pooled analysis of three trials. Oncology. 2012;83:241-247.

29. Tijink BM, Neri D, Leemans CR, et al. Radioimmunotherapy of head and neck cancer xenografts using ${ }^{131} \mathrm{I}$-labeled antibody L19-SIP for selective targeting of tumor vasculature. J Nucl Med. 2006;47:1127-1135.

30. Casey JL, Napier MP, King DJ, et al. Tumour targeting of humanised crosslinked divalent-fab[prime] antibody fragments: a clinical phase I/II study. $\mathrm{Br} \mathrm{J}$ Cancer. 2002;86:1401-1410.

31. Freireich EJ, Gehan EA, Rall DP, Schmidt LH, Skipper HE. Quantitative comparison of toxicity of anticancer agents in mouse, rat, hamster, dog, monkey, and man. Cancer Chemother Rep. 1966;50:219-244. 